\title{
The transformation of the human dimension in the cyberspace
}

\author{
Karamjit S. Gill
}

Published online: 24 July 2012

(C) Springer-Verlag London Limited 2012

In the very interdisciplinary tradition of AI \& Society, this issue covers a diversity of topics ranging from mindloading, 'moral subject' and human dignity and autonomy, organisation as a cognitive machine, the dilemma of boundaries between femininity and masculinity in the cyberspace, knowledge sharing for sustainable development, the emerging robotisation of the organisation, to cultural acceptance of robots in richly complex regions of the world. Other issues covered in this issue include dialogical framework of skill training, wealth adjustment in an artificial society and speech recognition.

On mind-uploading, the argument is that 'mind-uploading is a futuristic process that involves scanning brains and recording relevant information which is then transferred into a computer', in the sense of 'transfers of both human minds and identities from biological brains into computers'. The world of ubiquitous technology brings about a new challenge of what is to be human, for example, what we understand by 'moral subject', in other words the subject's unique metaphysical qualities of dignity and autonomy. The argument posited in this issue is that concept of 'human dignity underlies the foundation of many democratic systems, particularly in Europe as well as of international treaties, including the Universal Declaration of Human Rights. Digital agents, artificial organisms as well as new capabilities of the human agents related to their embeddedness in digital and biotechnological environments bring about an important transformation of the human self appraisal', especially from an ethical perspective.

It is interesting to note how technological culture is beginning to subscribe to the transformation of the human

K. S. Gill ( $\square)$

Professor Emeritus, University of Brighton, Brighton, UK

e-mail: kgillbton@yahoo.co.uk organisation into a cognitive machine. In the human-centred tradition of AI \& Society, it can be seen as a transformation of the human dimension from wisdom to calculation and then to being a cognitive machine. The argument put forward in this issue is that 'cognitive machines contribute to improving cognitive abilities in the organisation by extending people's rationally and decisionmaking capacity, and by reducing intra-individual and group dysfunctional conflict'. The increasing acceptance of the organisation in terms of a cognitive machine leads to robotisation of the organisation, raising issues of the cultural acceptance of the cognitive machine in diverse cultural regions of the world.

As we live in a culture of ubiquitous technology where 'everything can be commodified, measured and calculated and can be put in the competitive market for sale, detached from its roots and purpose', we face a dilemma and challenge of what is meant to be human. The world of cyberspace poses a further dilemma as to where the boundaries may lie "between femininity and masculinity both in terms of lifestyle and thought style'. The issue could be posed as how to 'redefine our humanness in terms of the changing nature of science, technology and their deeper impact on human life'. This means also to take note how 'on the one hand our being in the cyberspace opens up new and exciting horizons before us, on the other hand how we ourselves are changed and transformed in the process'. This reorientation of the human dimension also raises issues of how societies use symbols and signs to communicate information in the technological world we live in, and in what ways the communication media conveys a broad range of different kinds of messages within diverse cultural contexts.

In the globalising world, knowledge sharing is increasingly seen as a crucial factor in cultivating sustainable 
environments. The core of this sharing is the recognition of the need for social interfacing, in congruence with the ways practitioners share knowledge in complex systems. Knowledge sharing in the globalising economic world raises issues of the plurality of the creation, transfer and servicing of knowledge, which resides in diverse regions of the world. Regional economic innovation and growth may in this scenario reside in creating geographical clusters such as science cities, which act as a coherent source of knowledge for sustainability. 